\title{
Effectiveness of Aromatherapy and Music Distraction in Managing Pediatric Dental Anxiety: A Comparative Study
}

\author{
Jeswin James ${ }^{1}$, N Retnakumari ${ }^{2}$, Kannan Vadakkepurayil ${ }^{3}$, Anupam Kumar Thekkeveetil $^{4}$, Arun Tom $^{5}$
}

\begin{abstract}
Background: Managing a child's anxiety is most critical for the success of treatment in the pediatric dental setup. Present trend advocates the use of non-aversive behavior management techniques.

Aim and objective: To compare and evaluate the efficacy of aromatherapy using orange essential oil with that of music distraction in the management of anxious pediatric dental patients.

Design: One hundred and fifty children of the age 6-8 years were divided into three groups of 50 each and performed restorative treatment under aromatherapy, with music distraction, without aromatherapy, or music distraction, respectively. Venham's picture test and facial image scale (FIS), pulse rate, respiratory rate, and oxygen saturation were recorded before and after the procedure.

Results: Compared with controls, both the aromatherapy group and music distraction group showed significant posttreatment change in anxiety levels with respect to Venham's picture test and FIS, along with the reduction in pulse rate and respiratory rate. When compared between music distraction and aromatherapy, it did not yield statistical significance, though music distraction showed better results compared to aromatherapy. Conclusion: Both music distraction and aromatherapy using orange essential oil are effective in anxiety management of which music distraction presents a comparatively better result. Either these non-invasive techniques alone or a combination of both may be employed in the dental setup of pediatric dentists for molding cooperative patients.

Keywords: Aromatherapy, Dental anxiety, Facial image scale, Music distraction, Venham's picture test.

International Journal of Clinical Pediatric Dentistry (2021): 10.5005/jp-journals-10005-1911
\end{abstract}

\section{INTRODUCTION}

Dental anxiety has been identified as a major problem in children and is considered an obstacle in rendering quality dental care. It is "an abnormal fear or dread of visiting the dentist for preventive care or therapy and unwarranted anxiety over dental procedures". It has been reported that dental anxiety often resulting in increased levels of caries and behavioral management problems in children. ${ }^{2}$ In addition, they are found to have a defective oral function and oral esthetics. ${ }^{3}$

In pediatric dental setup, the sights (needles), drilling sounds, smells of cut dentin and eugenol, along with the sensation of a high frequency of vibration are usually anxiety provocation factors. ${ }^{4}$ To counteract the factors like sounds and smell which increases the anxiety levels of the pediatric patients, audio distraction and aromatherapy can be employed as non-aversive techniques in which patient listens to music and experience pleasant aroma during the dental procedure. Because of its success in medical settings and adult dental patients, many dentists believe that these techniques may be successful in the management of pediatric dental patients.

Few studies have been conducted to evaluate the success rate of using aromatherapy and music distraction in reducing the fear and anxiety of pediatric dental patients. None of the researchers have tried to compare the efficiency of these two non-invasive, inexpensive techniques.

\section{Materials and Methods}

The study was carried out in child patients of age group 6-8 years seeking restorative treatment in the pedodontics department.
${ }^{1}$ Department of Pedodontics, Sree Anjaneya Institute of Dental Sciences, Kozhikode, Kerala, India

${ }^{2-4}$ Department of Pedodontics, Government Dental College, Kozhikode, Kerala, India

${ }^{5}$ Department of Oral and Maxillofacial Pathology, Sree Anjaneya Institute of Dental Sciences, Kozhikode, Kerala, India

Corresponding Author: Jeswin James, Department of Pedodontics, Sree Anjaneya Institute of Dental Sciences, Kozhikode, Kerala, India, Phone: +91 9446617702, e-mail: drjeswinjames@gmail.com

How to cite this article: James J, Retnakumari N, Vadakkepurayil K, et al. Effectiveness of Aromatherapy and Music Distraction in Managing Pediatric Dental Anxiety: A Comparative Study. Int J Clin Pediatr Dent 2021;14(2):249-253.

Source of support: Nil

Conflict of interest: None

\section{Inclusion Criteria}

- Healthy children of age group 6-8 years visiting the department to undergo routine dental treatments like restoration with no previous dental experience.

- Children who are Frankel + ve or Frankel-ve in cooperation.

\section{Exclusion Criteria}

- Medically compromised and special children.

- Children with any respiratory tract illnesses or cardiac problems, common cold, allergy, and acute dental pain.

(0) Jaypee Brothers Medical Publishers. 2021 Open Access This article is distributed under the terms of the Creative Commons Attribution 4.0 International License (https://creativecommons.org/licenses/by-nc/4.0/), which permits unrestricted use, distribution, and non-commercial reproduction in any medium, provided you give appropriate credit to the original author(s) and the source, provide a link to the Creative Commons license, and indicate if changes were made. The Creative Commons Public Domain Dedication waiver (http://creativecommons.org/publicdomain/zero/1.0/) applies to the data made available in this article, unless otherwise stated. 


\section{Methods}

One hundred and fifty children between the age of 6 years and 8 years were selected from among patients who were seeking routine dental treatments like restoration. The anxious children were selected by following Frankel's behavior rating scale \{Frankel's positive (+) or negative (-)\} and were divided into three equal groups. The children in the first group $(n=50)$ were provided dental treatment under aromatherapy with orange essential oil. The second group of children $(n=50)$ were provided treatment employing music distraction. The third group of children (controls $=50$ ) were treated without employing aromatherapy or music distraction.

A separate treatment room had been setup to conduct the study. Informed consent was obtained from the parents of children who were participating in the study.

The natural essential oil of orange (Citrus aurantium) was used. An electric aroma diffuser was used to diffuse the essential oil, sufficient enough to create the pleasant smell of orange in the treatment room. All these actions were performed half an hour before the patient's arrival on aromatherapy days. In the second set, a music player was installed and melodious music was played during the entire treatment time. The treatments for children in the control group were scheduled on different days without employing music or aromatherapy.

Initially, the child's pulse rate and oxygen saturation rate were recorded using a pulse oximeter and respiratory rate was also recorded manually. The child was asked to point the images he/she felt resembling him/her at that moment in Venham's picture test and also from the five faces in the facial image scale (FIS). The scores were noted. The child was taken to the dental operatory room which was separately prepared for the study and was allowed some period of relaxation under music or aroma before the commencement of treatment. The restorative procedure was done in all children. After the administration of treatment, pulse rate, respiratory rate, and oxygen saturation were again recorded and the child was again asked to point the images in the anxiety scales.

\section{Statistical Analysis}

The changes in pre- and posttreatment mean anxiety levels were compared using the ANOVA test. The median changes in pre- and posttreatment levels of pulse rate, respiratory rate, and levels of oxygen saturation were compared using Kruskal-Wallis test. The change in posttreatment levels of anxiety levels between each study group was compared using Tukey's HSD test and the levels of pulse rate, respiratory rate, and oxygen saturation between these groups were compared using Mann-Whitney test.

\section{Results}

The subjects in the music distraction group and aromatherapy group showed considerable difference in median anxiety levels pre- and posttreatment according to Venham's picture test and FIS, in contrast to the unchanged levels among the control group subjects (Table 1).

The control group showed an increase in mean pulse rate and respiratory rate with a reduction in oxygen saturation levels after treatment. The music distraction group showed a reduction in mean pulse rate and respiratory rate with an increase in oxygen saturation levels after treatment when compared with the other two study groups. The aromatherapy group showed a reduction in mean pulse rate and respiratory rate with an increase in oxygen
Table 1: Median anxiety levels of different groups according to Venham's picture test and facial image scale

\begin{tabular}{llllll}
\hline & \multicolumn{2}{c}{ Venham's picture test } & & \multicolumn{2}{c}{ Facial image scale } \\
\cline { 2 - 3 } \cline { 5 - 6 } Group & Pre & Post & & Pre & Post \\
\hline Control group & 4 & 4 & 3 & 3 \\
$\begin{array}{l}\text { Music distraction } \\
\text { group }\end{array}$ & 4 & 0 & 4 & 1 \\
$\begin{array}{l}\text { Aromatherapy } \\
\text { group }\end{array}$ & 4 & 0 & 3 & 1 \\
\hline
\end{tabular}

saturation levels after treatment. But the change is less compared to the music distraction group (Table 2).

Venham's picture test and FIS showed significant changes in anxiety scores, while considering mean \pm SD among all the three study groups and computed using ANOVA test. Median changes in anxiety levels with respect to pulse rate, respiratory rate, and oxygen saturation levels among three study groups when tested the statistical significance using Kruskal-Wallis test showed significance in all parameters except oxygen saturation level (Table 3).

The control group vs music distraction group showed a significant statistical difference with respect to Venham's picture test, FIS when computed using Tukey's HSD test. Also, statistical significance is obtained with pulse rate, respiratory rate, and oxygen saturation level using Mann-Whitney test. The control group vs aromatherapy group showed significant statistical difference with respect to Venham's picture test, FIS when computed using Tukey's HSD test. Pulse rate and respiratory rate showed statistically significant change with Mann-Whitney test in contrast to the oxygen saturation level which is not significant. The aromatherapy group vs music distraction group did not show any statistically significant difference with respect to Venham's picture test and FIS when computed using Tukey's HSD test. Also, pulse rate, respiratory rate, and oxygen saturation levels too did not show any statistically significant difference when computed using Mann-Whitney test (Table 4).

\section{Discussion}

The current study compared two non-invasive techniques, i.e., aromatherapy and music distraction to alleviate anxiety levels of the pediatric patients with that of the control group. The effectiveness of the two techniques was measured using two subjective measures such as Venham's picture test and FIS, along with three objective measures including pulse rate, respiratory rate, and levels of oxygen saturation.

Venham's picture test and FIS are two reliable measures of self-portrayed anxiety in children. Investigations by Venham and Gaulin-Kremer implied the picture test to be a simple, yet valid and reliable index of the young child's response to situational stress. ${ }^{5}$ Children are given eight pairs of images that show various emotions and they are asked to select the ones that reflect their emotional state best. The images were given various that scores range from 0 (no anxiety) to 8 (very anxious). The test is easy to perform and only takes 1 or 2 minutes, which makes it one of the scales of choice for children. ${ }^{6}$ The FIS is a state measure of children's dental anxiety and comprises a row of five faces ranging from very happy to very unhappy (scores ranging from 1 to 5 ; 5 indicating the highest anxiety). It is a suitable measure for assessing state child dental anxiety in even very young children. ${ }^{7}$

Pulse rate was chosen for analysis because it is the simplest biological parameter to measure and also because an increase in 
Table 2: Pre- and posttreatment pulse rate, respiratory rate, and oxygen saturation (mean \pm SD) with respect to control group, music distraction group, and aromatherapy group

\begin{tabular}{|c|c|c|c|c|c|c|}
\hline \multirow[b]{2}{*}{ Group } & \multicolumn{2}{|c|}{ Pulse rate } & \multicolumn{2}{|c|}{ Respiratory rate } & \multicolumn{2}{|c|}{ Oxygen saturation } \\
\hline & Pre & Post & Pre & Post & Pre & Post \\
\hline Control & $98.4 \pm 12.74$ & $103.2 \pm 11.2$ & $21.8 \pm 2.06$ & $21.9 \pm 1.97$ & $97.72 \pm 1.25$ & $97.58 \pm 1.09$ \\
\hline Music group & $100.5 \pm 13.18$ & $91.02 \pm 10.2$ & $21.88 \pm 2.19$ & $20.24 \pm 1.38$ & $97.56 \pm 1.25$ & $98.08 \pm 1.12$ \\
\hline Aromatherapy & $99.2 \pm 12.23$ & $90.36 \pm 10.71$ & $22.06 \pm 2.71$ & $20.44 \pm 1.76$ & $97.84 \pm 1.25$ & $98.12 \pm 0.98$ \\
\hline
\end{tabular}

Table 3: Change in pre-treatment and posttreatment anxiety scores with respect to Venham's picture test, facial image scale, pulse rate, respiratory rate, and oxygen saturation

\begin{tabular}{lllll}
\hline & \multicolumn{3}{c}{ Group } \\
\cline { 2 - 4 } & $\begin{array}{l}\text { Control } \\
\text { Proup }\end{array}$ & $\begin{array}{l}\text { Music distraction } \\
\text { group }\end{array}$ & $\begin{array}{l}\text { Aromatherapy } \\
\text { group }\end{array}$ & p value \\
\hline Venham's picture test $^{\mathrm{a}}$ & $0.06 \pm 1.24$ & $-4.1 \pm 1.45$ & $-4.1 \pm 2.15$ & $<0.001$ \\
Facial image scale $^{\mathrm{a}}$ & $0.42 \pm 1.16$ & $-2.34 \pm 1.04$ & $-2.14 \pm 1.28$ & $<0.001$ \\
Pulse rate $^{\mathrm{b}}$ & $5(-4.22)$ & $-9(-43.17)$ & $-8(-41.5)$ & 0.0001 \\
Respiratory rate $^{\mathrm{b}}$ & $0(-2.2)$ & $-2(-6.1)$ & $-2(-6.0)$ & 0.0001 \\
Oxygen saturation $^{\mathrm{b}}$ & $0(-2.2)$ & $1(-3.5)$ & $0(-1.2)$ & 0.005 \\
\hline
\end{tabular}

$p<0.001$ is considered significant

${ }^{\text {a Mean }} \pm$ S.D, the $p$ value is computed using ANOVA test

${ }^{\mathrm{b}}$ Median (Min, Max), the $p$ value is computed using Kruskal-Wallis test

Table 4: Comparison between different groups based on the mean (a) and median (b) with respect to the difference in pre- and posttreatment change in anxiety levels (based on Venham's picture test and facial image scale) and pulse rate, respiratory rate, and oxygen saturation levels

\begin{tabular}{llllll}
\hline Group & Venham's picture test $^{a}$ & Facial image scale $^{a}$ & Pulse rate $^{b}$ & Respiratory rate $^{b}$ & Oxygen saturation $^{b}$ \\
\hline Control vs music distraction & $<0.0001$ & $<0.0001$ & $<0.0001$ & $<0.0001$ & 0.001 \\
Control vs aromatherapy & $<0.0001$ & $<0.0001$ & $<0.0001$ & $<0.0001$ & 0.012 \\
Aromatherapy vs music distraction & 1.00 & 0.66 & 0.87 & 0.66 & 0.16 \\
\hline
\end{tabular}

${ }^{a} p$ value is computed using Tukey's HSD test (Facial image scale and Venham's picture test)

${ }^{\mathrm{b}} p$ value is computed using Mann-Whitney test; $p<0.016$ is significant after adjusting for multiple comparison (pulse rate, respiratory rate, and oxygen saturation)

heart rate is the most common physiological indicator for anxiety and fear. It has been shown that this index is more in line with the anxiety experienced in dental visits than the other physiological parameters. $^{8}$

Respiratory rate was selected because the levels of individual anxiety tend to increase the rate of respiration. It is believed to be a result of the increased involuntary muscle contraction which results in the increased activation of respiratory muscles and the respiratory centers in the brain. ${ }^{9}$ Stress and anxiety can alter the respiratory rate, which in time, may alter oxygen saturation and/ carbon dioxide levels in the blood. ${ }^{10}$ Hence, measurement of oxygen saturation rate was also used to assess the anxiety levels in the present study.

\section{Anxiety Levels in Controls Pre- and Posttreatment}

Median anxiety levels pre- and posttreatment according to Venham's picture test and FIS showed unchanged levels among the control group subjects. The control group showed an increase in mean pulse rate and respiratory rate with a reduction in oxygen saturation levels after treatment. This may be attributed to the tactile sensation and anxiety triggered by dental treatment. Fear and anxiety increases the amount of perceived pain and causes an alarm reaction manifested by hypothalamus oriented vasoconstriction and dilatation as well as the release of more epinephrine and norepinephrine, causing heightened values of physiological parameters. ${ }^{11}$

\section{Control vs Aromatherapy Group}

Aromatherapy was found to be very effective in reducing anxiety levels of the children compared to the controls. Levels of anxiety reduction in the aromatherapy group were in accordance with the studies of Lehrner et al., Kritsidima et al. in elder dental patients waiting for a dental procedure. Lehrner et al. observed that women who were exposed to orange odor had a higher level of calmness. ${ }^{12}$ Kritsidima et al. observed that lavender scent reduces anxiety in dental patients though future visits were unaffected. $^{13}$

The reduction in pulse rate could be due to the interaction of essential oil with the parasympathetic nervous system to modulate anxiety. ${ }^{14}$ Parasympathetic activity is increased by $12 \%$ and sympathetic activity is decreased by $16 \%$ with orange oil. ${ }^{15}$ Hence, orange essential oil might have had a relaxant effect on respiration. The postoperative levels of oxygen saturation in the aromatherapy group did not show any significant change compared to the control group. It could be due to the subtle and insidious hypoxia due to inadvertent flexure of the neck, which might restrict the airway during dental manipulations and that resulted in insignificant change in levels of oxygen saturation. ${ }^{16}$ 


\section{Control vs Music Distraction Group}

The effect of music distraction is in accordance with the observations of Marwah et al., Jindal and Kaur when investigations were conducted within the same age group of the sample population. Marwah et al. ascertained that music distraction decreased anxiety levels in pediatric dental patients undergoing oral prophylaxis and restorative procedures, though not to a significant level. ${ }^{17}$ Jindal and Kaur observed that in children undergoing four dental visits music distraction decreased anxiety to a significant level during the restorative procedure visit ( $3 r d$ ) and invasive procedure visit (4th). ${ }^{18}$

Reduction in pulse rate in music distraction group is in accordance with earlier studies by Singh et al. ${ }^{19}$ Respiratory rate reduction in the music group is in accordance with the observations by lkonomidou et al. in patients scheduled to undergo gynecologic laparoscopy under general anesthesia. The reduction in levels of respiratory rate could be due to the anxiolytic effect of music which in turn reduces the increased rate of respiration which is related to anxiety. Music has been shown to affect the non-dominant side of the brain and give rise to the release of endorphins. ${ }^{20}$ Oxygen saturation levels after the procedure showed very mild but insignificant change with respect to the control group subjects.

\section{Aromatherapy Group vs Music Distraction Group}

Compared to the controls, the aromatherapy group and the music distraction showed significant change in posttreatment levels of all the biomarkers in the study except the levels of oxygen saturation. But the changes in posttreatment levels did not give any significance when compared between aromatherapy and music distraction. Even though the results of the present study did not show any statistical significance in posttreatment levels of biomarkers, the music distraction group showed better results with comparatively more reduction in the levels of pulse rate and respiratory rate and a slight increase in oxygen saturation levels compared to the aromatherapy group.

The action of music in reducing levels of anxiety is different from that of aromatherapy using essential oils. The music modulates noradrenergic (norepinephrine) neurons in the brain stem and midbrain, which regulate the autonomic response of heart rate, pulse, blood pressure, respiration, body temperature, skin conductance, and muscle tension, along with cholinergic and dopaminergic neurotransmission. Brain stem activation also mediates sensory and motor function through epinephrine, norepinephrine, and serotonin. ${ }^{21}$

In contrast to the said mechanism of reducing anxiety using music distraction, the aromatherapy using essential oil acts through the inhalation of scented oils which results in localization of the volatile molecules from oil in the lungs leading to the rapid diffusion of these molecules into the blood which causes brain activation via systemic circulation. Along with the brain activation, the molecules which bind to the olfactory receptors create an electrophysical response that reaches the brain. This reaction is expected to cause the activation of a neocortex, which has an effect on the perception of odors and reaches the limbic system regions including the amygdale and hypothalamus, the areas where the levels of hormone and emotions are controlled. Thus, the overall levels of anxiety and subjective biomarkers are maintained. ${ }^{4}$

Aromatherapy, being an inexpensive and non-invasive relaxant is currently used worldwide in the management of various anxiety and stress-related disorders, chronic pain, and depression. Music distraction can be a highly effective complementary method contributing to the traditional clinical procedures in pediatric dentistry. Success and acceptance of such non-invasive distraction methods in medical settings and adult patients are well established. Hence, the future prospects of these techniques in dental procedures, particularly in anxious child dental patients should be taken up for further investigations.

\section{Conclusion}

- The control group showed an increase in posttreatment anxiety levels when measured using Venham's picture test and FIS. The posttreatment levels of pulse rate and respiratory rate were increased, with a reduction in levels of oxygen saturation.

- The music distraction group and aromatherapy group showed a significant reduction in anxiety levels compared to the controls when measured using Venham's picture test and FIS. Also, a significant reduction in posttreatment levels of pulse rate and respiratory rate with a slight increase in levels of oxygen saturation.

-When compared between aromatherapy and music distraction, the posttreatment levels did not yield statistical significance. Music distraction showed a better result with a comparatively more reduction in levels of pulse rate and respiratory rate and a meager increase in levels of oxygen saturation.

From the present study, it can be concluded that music distraction and aromatherapy, or a combination of both can be employed as behavior management techniques in the pediatric dental clinic to alleviate the anxiety of the child patient and making the dental visit a pleasant experience for the patient, parent, and the dental practitioner.

\section{References}

1. Encyclopedia of medical concepts. National Library of Medicine, Ontario, Canada; 2009. (Cited 2010 June 4). Available from http:// www.reference.md/files/D016/mD016854.html.

2. Hollis A, Willcoxson $F$, Smith $A$, et al. An investigation into dental anxiety amongst paediatric cardiology patients. Int J Paediatr Dent 2015;25(3):183-190. DOI: 10.1111/ipd.12111.

3. Schuller A, Willumensen T, Holst D. Are there differences in oral health and oral health behavior between individuals with high and low dental fear? Community Dent Oral Epidemiol 2003;31(2):116-121. DOI: 10.1034/j.1600-0528.2003.00026.x.

4. Jafarzadeh M, Arman S, Pour FF. Effect of aromatherapy with orange essential oil on salivary cortisol and pulse rate in children during dental treatment: a randomized controlled clinical trial. Adv Biomed Res 2013;2(1):10. DOI: 10.4103/2277-9175.107968.

5. Venham LL, Gaulin-Kremer E. A self-report measure of situational anxiety for young children. Pediatr Dent 1979;1(2):91-96.

6. Jimeno FG, Bielsa SY, Fernandez CC, et al. Objective and subjective measures for assessing anxiety in paediatric dental patients. Eur J Paediatr Dent 2011;12(4):239-244.

7. Buchanan $\mathrm{H}$, Niven $\mathrm{N}$. Validation of a facial image scale to assess child dental anxiety. Int J Paediatr Dent 2002;12(1):47-52. DOI: 10.1046/j.0960-7439.2001.00322.x.

8. Shindova M, Belcheva A. The effect of parental presence on the dental anxiety during clinical examination in children aged 6-12 years. J IMAB 2013;19(4):435-438. DOI: 10.5272/jimab.2013194.435.

9. Masaoka Y, Homma I. The effect of anticipatory anxiety on breathing and metabolism in humans. Respirat Physiol 2001;128(2):171-177. DOI: 10.1016/s0034-5687(01)00278-x.

10. Padma R, Goel S, Shriniwas M, et al. Comparative evaluation of the oxygen saturation levels using pulse oxiemer during non-surgical and 
surgical periodontal therapy. J Contemp Dent Pract. 2012;13(5):661664. DOI: 10.5005/jp-journals-10024-1205.

11. Sanadhya $Y$, Sanadhya S, Jalihal S, et al. Hemodynamic, ventilator, and ECG changes in pediatric patients undergoing extraction. J Indian Soc Pedod Prev Dent. 2013;31(1):10-16. DOI: 10.4103/0970-4388. 112393.

12. Lehrner J, Eckersberger C, Walla P, et al. Ambient odor of orange in a dental office reduces anxiety and improves mood in female patients. Physiol Behav 2000;71(1-2):83-86. DOI: 10.1016/s0031-9384(00) 00308-5.

13. Kritsidima M, Newton T, Asimakopoulou K. The effects of lavender scent on dental patient anxiety levels: a cluster randomisedcontrolled trial. Community Dent Oral Epidemiol 2010;38(1):83-87. DOI: 10.1111/j.1600-0528.2009.00511.x.

14. Chien LW, Cheng SL, Liu CF. The effect of lavender aromatherapy on autonomic nervous system in midlife women with Insomnia. Evid Based Complement Alternat Med 2012;2012:740813. DOI: 10.1155/2012/740813.
15. Dobetsberger C, Buchbauer G. Actions of essential oils on the central nervous system: an updated review. Flavour Fragrance J 26(5):300-316. DOI: 10.1002/ffj.2045.

16. Bello LL, Darwish SK. Effect of restorative dental procedures on vital signs in children. Saudi Dent J 6(2):78-82.

17. Marwah N, Prabhakar AR, Raju OS. Music distraction--its efficacy in management of anxious pediatric dental patients. J Indian Soc Pedod Prev Dent. 2005;23(4):168-170. DOI: 10.4103/0970-4388.19003.

18. Jindal R, Kaur R. Can we tune our pediatric patients? Int J Clin Pediat Dentis 2011;4(3):186-189. DOI: 10.5005/jp-journals-10005-1107.

19. Singh D, Samadi F, Jaiswal J, et al. Stress reduction through audio distraction in anxious pediatric dental patients: an adjunctive clinical study. Int J Clin Pediat Dentis 2014;7(3):149-152. DOI: 10.5005/ jp-journals-10005-1254.

20. Ikonomidou E, Rehnstrom A, Naesh O. Effect of music on vital signs and postoperative pain. Aorn J 2004;80(2):269-274. DOI: 10.1016/ s0001-2092(06)60564-4, 277-8.

21. Chanda ML, Levitin DJ. The neurochemistry of music. Trends Cognit Sci 17(4):179-193. DOI: 10.1016/j.tics.2013.02.007. 